\title{
The filtering analog of the variational multiscale method in large-eddy simulation
}

\author{
A. W. Vreman a) \\ Numerical Analysis and Computational Mechanics, Faculty EWI, University of Twente, P.O. Box 217, \\ 7500 AE Enschede, The Netherlands
}

(Received 9 January 2003; accepted 14 April 2003; published 2 July 2003)

\begin{abstract}
The variational multiscale method introduced by Hughes et al. [Comput. Visual. Sci. 3, 47 (2000)] is extended to the classic filtering approach in large-eddy simulation. The role of the Germano identity in the formulation is precisely indicated. Multiscale methods based on standard eddy-viscosity models are related to (anisotropic) hyperviscosity models under certain conditions. Several models are tested and found to be as accurate as the standard dynamic model, while the implementations are more simple. Finally, the turbulent stress tensor is reformulated, such that filter and derivative in the filtered equations can be treated as a single operator. (C) 2003 American Institute of Physics. [DOI: 10.1063/1.1595102]
\end{abstract}

The variational multiscale method (VMS) proposed by Hughes et al. ${ }^{1-3}$ and clarified by Collis ${ }^{4}$ is a promising approach to the large-eddy simulation (LES) of turbulent flows. It appeared that the Smagorinsky model, without wall functions normally not accurate in wall bounded and transitional flows, improved considerably when applied in a multiscale context and became at least as accurate as the dynamic model. ${ }^{2,3}$ In VMS three classes of scales are considered: ${ }^{4}$ large, small and unresolved. The first two classes are solved with LES, whereas the unresolved scales are modeled. Two modeling assumptions for the effect of the unresolved scales are used in Refs. 1-4: (a) it is neglected in the large-scale equation and (b) it is modeled in the small-scale equation, with a standard LES model, but expressed in the small scales.

In Refs. 1-4 the method is formulated as a variational approach, i.e., posed in a weak formulation involving the multiplication with test functions. A key feature of VMS is the projection operator which separates scales using a set of basis functions and this has several mathematical advantages. ${ }^{1-4}$ A specific case of the variational formulation is the Fourier-Galerkin method., ${ }^{2,3}$ Fourier methods are nonlocal and therefore not applicable in complex flows. However, VMS can be applied to complex flows with use of, for example, finite element methods or discontinuous Galerkin methods. ${ }^{1,4}$

In this Letter, VMS is extended to the filtering approach and then the classic approach for LES of complex flows is followed, which is based on the application of a filter to the Navier-Stokes equations, e.g., the top-hat filter, together with finite difference or finite volume discretizations. Filters in LES (except the spectral cutoff) are not projections but smoothing operators. For each function a large and a smallscale component can precisely be defined, but these components do not live in disjunct function spaces.

Filtering multiscale methods have been considered be-

${ }^{a)}$ Electronic mail: a.w.vreman@utwente.nl fore to some extent, such as a more complicated approach which involves computations on multiple grids. ${ }^{6}$ Jeanmeart and Winckelmans ${ }^{7}$ tested a specific model using a discrete compact filter in a partially spectral computation. In this Letter we will go further: other models will be considered, the basic filtering multiscale equations with their subgrid terms will be formulated, and finite differencing in all directions will be used to test the models.

The standard filtered momentum equation in LES reads

$$
\partial_{t} \bar{u}_{i}+\partial_{j}\left(\bar{u}_{i} \bar{u}_{j}\right)+\partial_{j} \tau_{i j}=-\partial_{i} \bar{p}+\partial_{j} \bar{\sigma}_{i j},
$$

where $u$ is the velocity, $p$ the pressure and $\sigma$ viscous stress tensor. The partial derivatives $\partial / \partial t$ and $\partial / \partial x_{j}$ are denoted with $\partial_{t}$ and $\partial_{j}$, respectively. The bar denotes an arbitrary filter with filter width $\bar{\Delta}$ extracting the resolved $\bar{u}$ from the original $u$. Furthermore, $\tau_{i j}=\overline{u_{i} u_{j}}-\bar{u}_{i} \bar{u}_{j}$ is the turbulent stress tensor.

In order to split the resolved scales in a large- and a small-scale part, a second filter with filter width $\hat{\Delta}$ is introduced, a so-called test filter. ${ }^{8}$ The large-scale component of a quantity $f$ is denoted with $\hat{f}$ and the small-scale component is defined by ${ }^{7,8}$

$$
f^{\prime}=f-\hat{f} .
$$

In this paper the operation denoted with a prime is called the small-scale extraction. For the large and small-scale parts of the resolved velocity $\bar{u}$, simplified notations are introduced:

$$
V=\hat{\bar{u}} ; \quad v=\bar{u}^{\prime}=\bar{u}-\hat{\bar{u}}=\bar{u}-V .
$$

Next we write the exact equations for the large and for the small scales. The turbulent stress for the large scales is defined as

$$
T_{i j}=\widehat{\overline{u_{i} u_{j}}}-V_{i} V_{j}
$$

The large-scale equation then reads

$$
\partial_{t} V_{i}+\partial_{j}\left(V_{i} V_{j}\right)+\partial_{j} T_{i j}=-\partial_{i} \hat{\bar{p}}+\partial_{j} \hat{\bar{\sigma}}_{i j},
$$


and when this equation is subtracted from (1) the small-scale equation is obtained:

$$
\begin{aligned}
\partial_{t} v_{i} & +\partial_{j}\left(\bar{u}_{i} \bar{u}_{j}\right)-\partial_{j}\left(V_{i} V_{j}\right)+\partial_{j} \tau_{i j}-\partial_{j} T_{i j} \\
& =-\partial_{i} \bar{p}^{\prime}+\partial_{j} \bar{\sigma}_{i j}^{\prime} .
\end{aligned}
$$

To rewrite these equations, the Germano identity is very useful:

$$
T_{i j}=\hat{\tau}_{i j}+L_{i j},
$$

where the so-called resolved turbulent stress is defined by

$$
L_{i j}={\widehat{\overline{u_{i}}}}_{j}-\hat{\bar{u}}_{i} \hat{\bar{u}}_{j}=\widehat{\bar{u}}_{i} \bar{u}_{j}-V_{i} V_{j} \text {. }
$$

Substituting (7) into (5) and (6) yields

$$
\begin{aligned}
& \partial_{t} V_{i}+\partial_{j}\left(V_{i} V_{j}\right)+\partial_{j} L_{i j}+\partial_{j} \hat{\tau}_{i j}=-\partial_{i} \hat{\bar{p}}+\partial_{j} \hat{\bar{\sigma}}_{i j}, \\
& \partial_{t} v_{i}+\partial_{j}\left(\bar{u}_{i} \bar{u}_{j}-\widehat{\bar{u}}_{i} \bar{u}_{j}\right)+\partial_{j}\left(\tau_{i j}-\hat{\tau}_{i j}\right)=-\partial_{i} \bar{p}^{\prime}+\partial_{j} \bar{\sigma}_{i j}^{\prime} .
\end{aligned}
$$

The last equation equals

$$
\partial_{t} v_{i}+\partial_{j}\left(\bar{u}_{i} \bar{u}_{j}\right)^{\prime}+\partial_{j} \tau_{i j}^{\prime}=-\partial_{i} \bar{p}^{\prime}+\partial_{j} \bar{\sigma}_{i j}^{\prime} .
$$

Finally, the sum of (9) and (11) provides the equation that needs to be modeled in "filtering multiscale LES:"

$$
\partial_{t} \bar{u}_{i}+\partial_{j}\left(\bar{u}_{i} \bar{u}_{j}\right)+\partial_{j} \hat{\tau}_{i j}+\partial_{j} \tau_{i j}^{\prime}=-\partial_{i} \bar{p}+\partial_{j} \bar{\sigma}_{i j} .
$$

This might appear to be a trivial result, but its derivation is important, because Eq. (9) identifies $\hat{\tau}_{i j}$ as the subgrid term in the large-scale equation and Eq. (11) identifies $\tau_{i j}^{\prime}$ as subgrid term in the small scale equation. The knowledge of the origin of $\hat{\tau}$ and $\tau^{\prime}$ can be used to model its sum $\tau$. The modeling assumptions in VMS according to Refs. $1-4$ are: (a) to neglect $\hat{\tau}_{i j}$ in the weak form the large-scale equation, which implies by analogy $\hat{\tau}_{i j}=0$ in Eq. (9), and (b) to model $\tau_{i j}^{\prime}$ with (for example) the Smagorinsky model in terms of $v$. In the following, we will discuss assumption (b) first and then assumption (a).

Now we turn to the modeling of $\tau_{i j}^{\prime}$ based on, e.g., the Smagorinsky model,

$$
\begin{aligned}
& m_{i j}(\bar{u})=-\nu_{e}(\bar{u}) S_{i j}(\bar{u}), \\
& \nu_{e}(\bar{u})=C_{S}^{2} \bar{\Delta}^{2} S(\bar{u}) ; \quad S=\left(\frac{1}{2} S_{i j} S_{i j}\right)^{1 / 2} .
\end{aligned}
$$

Three options to construct a model for $\tau_{i j}^{\prime}$ using $m_{i j}$ are considered:

(M1) The small-scale extraction from $m_{i j}(\bar{u})$,

$$
\tau_{i j}^{\prime}=\left(m_{i j}(\bar{u})\right)^{\prime} .
$$

(M2) Model $m_{i j}$ expressed in the small-scale velocity,

$$
\tau_{i j}^{\prime}=m_{i j}(v) \text {. }
$$

(M3) The small-scale extraction from M2,

$$
\tau_{i j}^{\prime}=\left(m_{i j}(v)\right)^{\prime} .
$$

The last model uses two small-scale restrictions; it is first based on $v$ instead of $\bar{u}$ and then the small-scale operator ( )' is applied. Perhaps, both restrictions are not simultaneously needed, reason to introduce models 1 and 2. Model 3 was proposed by Hughes et al. ${ }^{1-3}$ (but then in the variational formulation) as the "small-small" model. It is very interesting that the classic paper by Schumann ${ }^{9}$ contained a special case of multiscale model 2: the Smagorinsky model is applied to the strain rate without its mean, i.e., the test filter is the ensemble average. The "large-small" models in Refs. 1-3 and 7 are somewhat different from M1-M3, because they express the strain rate in $v$, but the eddy viscocity in $\bar{u}$.

We proceed to argue that all these models in combination with a top-hat or Gaussian filter are related to (anisotropic) hyperviscosity mechanisms. The first term in the Taylor expansion ${ }^{10}$ of the small-scale quantity is an "anisotropic" Laplacian operator:

$$
f^{\prime}=-\frac{1}{24}\left(\widehat{\Delta}_{1}^{2} \partial_{1}^{2} \bar{f}+\widehat{\Delta}_{2}^{2} \partial_{2}^{2} \bar{f}+\widehat{\Delta}_{3}^{2} \partial_{3}^{2} \bar{f}\right) .
$$

For differential filters this equation is exact. ${ }^{11}$ For constant $\nu_{e}$ and incompressible flow, the eddy-viscosity model (13) reduces to a Laplacian. Under the same conditions and with Eq. (18), models M1 and M2 become proportional to double Laplacians (fourth-order dissipations), and M3 becomes proportional to a triple Laplacian (sixth-order dissipation).

The variational multiscale models (with orthogonal projection) were proven to dissipate kinetic energy. ${ }^{1}$ This is also a precise analytic property of the filtering multiscale model M3, under some conditions. A filter is symmetric if the filter kernel satisfies $G(x, \xi)=G(\xi, x)$. By substitution of the filter definition, it is easy to prove that for a symmetric test filter

$$
\int \hat{g}=\int \hat{f} g \text { and } \int f g^{\prime}=\int f^{\prime} g .
$$

Consequently, M3 inherits the dissipative character of $m_{i j}$, provided the test filter is not only symmetric but also commutes with derivatives:

$$
\epsilon_{M 3}=\int \bar{u}_{i} \partial_{j}\left(m_{i j}(v)\right)^{\prime}=\int v_{i} \partial_{j} m_{i j}(v) \geqslant 0 .
$$

With some calculation, model M1 can also be proven to be dissipative, at least for the Smagorinsky base model, using Eq. (18) and constant filter widths:

$$
\epsilon_{M 1}=\int C_{S}^{2} \bar{\Delta}^{2} S \hat{\Delta}_{k}^{2}\left(\left(\partial_{k} S\right)^{2}+\frac{1}{2}\left(\partial_{k} S_{i j}\right)^{2}\right) \geqslant 0 .
$$

The size of the test filter controls the activity of the small-scale model. For small $\hat{\Delta}$ the models for $\tau_{i j}^{\prime}$ are relatively small, while for large $\hat{\Delta}$ they approach the basic model $m_{i j}$. There may be cases that the modeling assumption $\hat{\tau}_{i j}$ $=0$ in the large-scale equation is not true (for example when the test filter is very small) and then mixed models seem natural candidates to model $\tau_{i j}$.

Assume for example a similarity or gradient (=nonlinear) model $\alpha_{i j}$ for $\tau_{i j}$, which correlate well with $\tau_{i j} \cdot{ }^{12}$ Since the small-scale dissipation of these models is inadequate, they could be proposed to model the large-scale turbulent stress $\hat{\tau}_{i j}$ only. If the small-scale part $\tau_{i j}^{\prime}$ is modeled by $\beta_{i j}$ provided by $\mathrm{M} 1-\mathrm{M} 3$, a mixed model reads: $\tau_{i j}=\hat{\alpha}_{i j}$ $+\beta_{i j}$. If the size of the test filter increases, $\hat{\alpha}$ becomes smaller and $\beta$ becomes larger. Other options for the closure of the large-scale equation were listed in Ref. 4.

In summary, multiscale LES simulates Eq. (1) and only alters the modeling of $\tau$, which is decomposed as $\tau=\hat{\tau}$ 


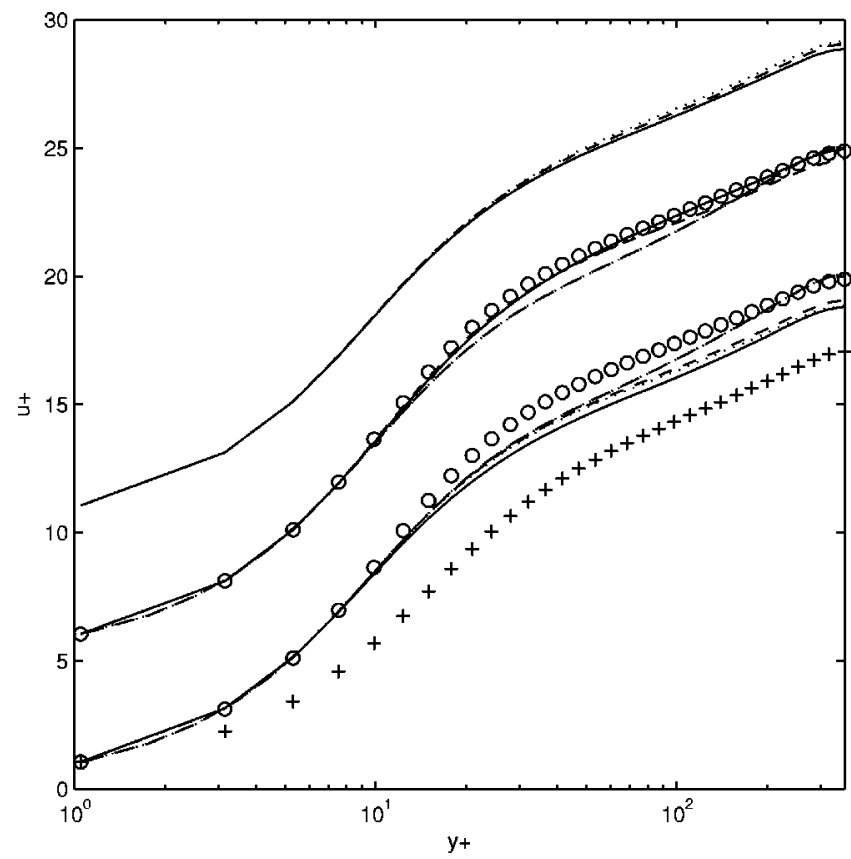

FIG. 1. Three sets of mean flow profiles. Lowest set: Smagorinsky model (+), M1 (○), M2 (dashed line), M3 (dotted line), no-model LES (solid line) and DNS from Ref. 14 (dashed-dotted line). Middle set $(5+u)$ : M1 $(\bigcirc)$, M2 $\left(C_{S}=0.2\right.$, dashed line), dynamic model (solid line) and DNS (Ref. 14) (dashed-dotted line). Highest set $(10+u)$ : M2 (dashed line), M2 plus filtered gradient (dotted line) and M2 plus filtered similarity (solid line).

$+\tau^{\prime} . \tau^{\prime}$ is the subgrid term that occurs in the small-scale equation. It is modeled with, e.g., M1-M3, related to hyperviscosities. $\hat{\tau}$ is the subgrid term in the large-scale equation, which is either neglected or modeled. The latter case results in a mixed model for $\tau$, where the test-filter size defines the level of activity of $\tau$ 's components.

Tests are performed for a turbulent channel flow with $\operatorname{Re}_{\tau}=360$ in a domain of size $6 H \times 2 H \times 2 H$ on a collocated $48 \times 63 \times 48$ grid, using a second-order energy-conserving finite difference method. The test case is somewhat similar to Ref. 13. The LES results are compared with DNS results taken from www.afm.ses.soton.ac.uk/ zhi/channeldata. ${ }^{14}$ The top-hat test filter is applied in three directions and approximated with the trapezoidal rule using $\widehat{\Delta}_{i}=2 \bar{\Delta}_{i}=2 h_{i}$. In addition the test filter is not allowed to cross wall boundaries. $C_{S}=0.1$ unless a different value is indicated.

The lowest set of mean flow profiles in Fig. 1 shows that the three models M1-M3 are closer to the DNS data ${ }^{14}$ than the no-model case and the Smagorinsky model. In more detail, M1 overpredicts the velocity in the near-wall region, while M2 and M3 underpredict the velocity in the center of the channel. For the same $C_{S}$, M1 has more effect than M2, while M2 has more effect than M3.

The middle set of curves in Fig. 1 demonstrates that the relatively simple multiscale models are as accurate as the standard dynamic model. This conclusion is supported by the Reynolds stress predictions, for which an example is shown in Fig. 2. The optimal value of $C_{S}$ in M2 and M3 appears to be closer to 0.2 than to 0.1 (see also Ref. 2). The highest sets of curves include results for the mixed model $\hat{\alpha}+\beta$, where $\beta$ equals M2 and $\alpha$ equals either $\tau(\bar{u})$ or $\frac{1}{12} \Delta_{k}^{2} \partial_{k} \bar{u}_{i} \partial_{k} \bar{u}_{j}$. In-

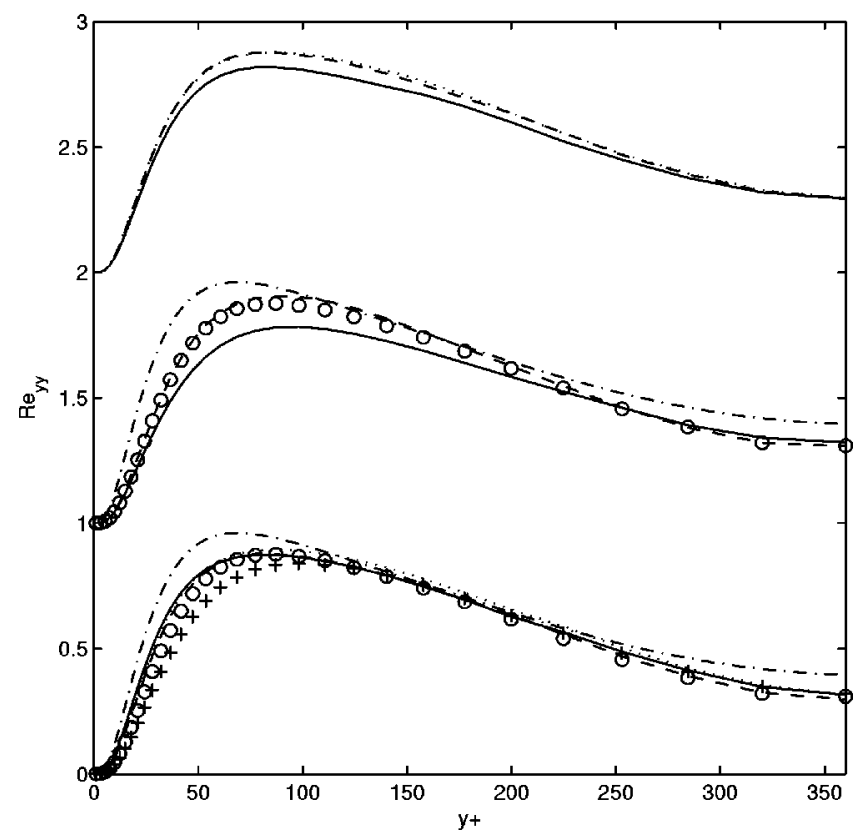

FIG. 2. Reynolds stress profiles $\mathrm{Re}_{y y}$. See caption of Fig. 1 .

clusion of the similarity and gradient components does not further improve the results, but this could be different for another test-filter width or in another flow.

The formulation so far was based on (1), the equation used in standard LES. In the following the filtered equations are rewritten such that each term is an analog of a Galerkin projection,

$$
\partial_{t} \bar{u}_{i}+\overline{\partial_{j}\left(\bar{u}_{i} \bar{u}_{j}\right)}+\overline{\partial_{j} a_{i j}}=-\overline{\partial_{i} p}+\overline{\partial_{j} \sigma_{i j}}
$$

with a redefined turbulent stress $a_{i j}=u_{i} u_{j}-\bar{u}_{i} \bar{u}_{j}$. The same equation actually occurred in Ref. 10 , with the notable difference that we do not interchange filter and derivative. Now there is no commutation required between partial derivatives and filter operation, at least not for the convective terms. The absence of commutation errors was claimed to be an important advantage of VMS. ${ }^{1-4}$

Equation (22) can be solved with the explicit filtering technique, but it is more attractive to treat the spatial filter and derivative as one operator, i.e., to discretize $\overline{\partial_{j} f}$ directly. A well-known example is the Fourier-Galerkin method in combination with a spectral cutoff filter. For complex flows, the combination of top-hat filter and spatial derivative suggests a finite volume method, because Gauss' theorem reduces the filter volume integral of a derivative to a difference of two surface integrals. ${ }^{9,15}$

Again (alternative) equations for $V$ and $v$ can be derived,

$$
\begin{aligned}
& \partial_{t} V_{i}+\widehat{\widehat{\partial_{j} V_{i} V_{j}}}+\widehat{\widehat{\partial_{j} B_{i j}}}+\widehat{\widehat{\partial_{j} a_{i j}}}=\widehat{\widehat{\mathrm{rhs}}}, \\
& \partial_{t} v_{i}+\left(\overline{\partial_{j} \bar{u}_{i} \bar{u}_{j}}\right)^{\prime}+\left(\overline{\partial_{j} a_{i j}}\right)^{\prime}=\mathrm{rhs}^{\prime} .
\end{aligned}
$$

The trivial identity $A_{i j}-a_{i j}=B_{i j}$ is used, where $A_{i j}=u_{i} u_{j}$ $-V_{i} V_{j}$ is the turbulent stress on the large-scale level and $B_{i j}=\bar{u}_{i} \bar{u}_{j}-V_{i} V_{j}$ is a resolved stress. ${ }^{15}$ The formulation 
based on Eq. (1) is only an analog of VMS, but Eqs. (23) and (24) reduce to an equivalence of VMS in case the filter is a projection in a Galerkin method.

Denoting the combined filter and partial derivative with $\delta_{j}$, i.e., $\delta_{j} f=\overline{\partial_{j} f}$, the convective term in Eq. (22) equals $\delta_{j}\left(\bar{u}_{i} \bar{u}_{j}\right)$. The subgrid-term then equals $\delta_{j} a_{i j}$. It is split into large- and small-scale components, $\widehat{\delta_{j} a_{i j}}$ and $\left(\delta_{j} a_{i j}\right)^{\prime}$. The large-scale component is precisely the subgrid term in (23). It could be neglected, or a similarity-gradient model derived from the definition of $a_{i j}$ could be used. ${ }^{15}$ The small-scale component is modeled by $\left(\delta_{j} m_{i j}\right)^{\prime}$, where $m_{i j}$ is based on $\bar{u}$ or $v$.

\section{ACKNOWLEDGMENTS}

The author thanks F. van der Bos, H. Kuerten, B. Geurts, J. van der Vegt, N. Sandham and R. Verstappen for relevant literature and suggestions about how to set up a channel flow simulation.

${ }^{1}$ T.J.R. Hughes, L. Mazzei, and K.E. Jansen, "Large eddy simulation and the variational multiscale method," Comput. Visual. Sci. 3, 47 (2000).

${ }^{2}$ T.J.R. Hughes, L. Mazzei, A.A. Oberai, and A.A. Wray, "The multiscale formulation of large eddy simulation: Decay of homogeneous isotropic turbulence," Phys. Fluids 13, 505 (2001).

${ }^{3}$ T.J.R. Hughes, A.A. Oberai, and L. Mazzei, "Large eddy simulation of turbulent channel flows by the variational multiscale method," Phys. Fluids 13, 1784 (2001).
${ }^{4}$ S.S. Collis, "Monitoring unresolved scales in multiscale turbulence modeling," Phys. Fluids 13, 1800 (2001).

${ }^{5}$ T. Dubois, F. Jauberteau, and R. Temam, "Incremental unknowns, multilevel methods and the numerical simulation of turbulence," Comput. Methods Appl. Mech. Eng. 159, 124 (1998).

${ }^{6}$ M. Terracol, P. Sagaut, and C. Basdevant, "A multilevel algorithm for large-eddy simulation of turbulent compressible flows," J. Comput. Phys. 167, 439 (2001).

${ }^{7}$ H. Jeanmeart and G.S. Winckelmans, "Comparison of recent dynamic subgrid-scale models in turbulent channel flow," in Studying Turbulence Using Numerical Simulation Databases IX (CTR, Stanford, 2002), pp. 105-116.

${ }^{8}$ M. Germano, U. Piomelli, P. Moin, and W.H. Cabot, "A dynamic subgridscale model," Phys. Fluids A 3, 1760 (1991).

${ }^{9}$ U. Schumann, "Subgrid scale model for finite difference simulations of turbulent flows in plane channels and annuli," J. Comput. Phys. 18, 376 (1975).

${ }^{10}$ A. Leonard, "Energy cascade in large-eddy simulations of turbulent fluid flows," Adv. Geophys. 18, 237 (1974).

${ }^{11} \mathrm{M}$. Germano, "Differential filters for the large eddy numerical simulation of turbulent flows," Phys. Fluids 29, 1755 (1986).

${ }^{12} \mathrm{C}$. Meneveau and J. Katz, "Scale-invariance and turbulence models for large-eddy simulation," Annu. Rev. Fluid Mech. 32, 1 (2000).

${ }^{13}$ Y. Morinishi and O.V. Vasilyev, "Vector level identity for dynamic subgrid scale modeling in large eddy simulation," Phys. Fluids 14, 3616 (2002).

${ }^{14}$ Z.W. Hu and N.D. Sandham, "DNS databases for turbulent Couette and Poiseuille flow," Report AFM-01/04, University of Southampton (2001).

${ }^{15}$ A.W. Vreman and B.J. Geurts, "A new treatment of commutation-errors in LES," in Advances in Turbulence IX, edited by I.P. Castro, P.E. Hancock, and T.G. Thomas (CIMNE, Barcelona, 2002), pp. 199-202. 